\title{
ECONOMIA PSÍQUICA DOS ALGORITMOS E LABORATÓRIO DE PLATAFORMA: MERCADO, CIÊNCIA E MODULAÇÃO DO COMPORTAMENTO
}

PSYCHIC ECONOMY OF ALGORITHMS AND PLATFORM LABORATORY:

MARKET, SCIENCE AND BEHAVIOR MODULATION

ECONOMÍA PSIQQUICA DE LOS ALGORITMOS Y LABORATORIO

DE PLATAFORMAS: MERCADO, CIENCIA Y MODULACIÓN DEL COMPORTAMIENTO

Fernanda Glória Bruno'

Anna Carolina Franco Bentes ${ }^{2}$

Paulo Faltay ${ }^{3}$

RESUMO: o extensivo e ininterrupto monitoramento de nossas ações online integram as engrenagens de um investimento econômico que direciona imensos volumes de dados para aplicação de estratégias de modificação do comportamento humano. Nesse contexto, observamos um aumento do interesse tecnocientífico, econômico e social em processos algorítmicos de extração e utilização de dados psíquicos e emocionais. Este artigo tem como objetivo apresentar reflexões sobre essa Economia Psíquica dos Algoritmos, enfatizando sua inquietante dimensão laboratorial. Analisando casos recentes e aspectos da arquitetura das plataformas digitais, mostraremos como o uso desses dados articula três camadas: a econômica; a epistemológica; e a de gestão e controle comportamental. Notaremos que o cruzamento dessas camadas tornam as fronteiras

\footnotetext{
ORCID: 0000-0002-5950-7602 - e-mail: bruno.fernanda@gmail.com

ORCID: 0000-0003-4167-6093 - e-mail: annacbentes@gmail.com

ORCID: 0000-0002-2467-1357 - e-mail: pfaltay@gmail.com
} 
entre o laboratório e a vida social extremamente tênues, o que nos convoca a recolocar o problema das relações entre ciência, tecnologia e sociedade nesse novo cenário.

Palavras-Chave: Algoritmos. Laboratório de Plataforma. Subjetividade.

ABSTRACT: the extensive and uninterrupted tracking of our online actions integrates the mechanism of an economic investment that employs data-driven human behavior modification strategies. In this context, we observe an increase of the technoscientific, economic and social interest in algorithmic processes based on the use of psychic and emotional information. This article aims to present insights on this Psychological Economics of Algorithms, emphasizing its disturbing laboratory dimension. Analyzing recent cases and aspects of digital platform architecture, we will show how the use of this data connects three layers: the economic one; the epistemological; and the behavioral management and control. We will note that the intersection of these layers make the boundaries between the laboratory and social life extremely blurred, which displays the troubled relations between science, technology and society in this emerging scenario.

Keywords: Algorithms. Plattform Laboratory. Subjectivity.

RESUMEM: el monitoreo amplio y continuo de nuestras acciones online integra los engranajes de una inversión económica que hace uso de inmensos volúmenes de datos para aplicación de estrategias de modificación del comportamiento. En este contexto, observamos un aumento de interés tecnocientífico, económico y social en los procesos algorítmicos de uso de informaciones psíquicas y emocionales. Este artículo presenta reflexiones sobre esta Economía Psíquica de los Algoritmos, haciendo hincapié en su inquietante dimensión de laboratorio. Analizando casos recientes y aspectos de la arquitectura de la plataforma digital, mostraremos cómo el uso de estos datos articula tres capas: la económica; la epistemológica; la de gestión y control comportamental. Notaremos que la intersección de estas capas vuelve los límites entre el laboratorio y la vida social extremadamente borrosos, llamándonos a reubicar el problema de las relaciones entre ciencia, tecnología y sociedad en este nuevo escenario.

Palabras Clave: Algoritmos. Laboratorio de plataformas. Subjetividad. 


\section{Introdução}

Nossos smartphones são um questionário psicológico que está sendo constantemente preenchido, tanto consciente quanto inconscientemente.

Em maio de 2017, um relatório interno produzido por dois executivos da filial australiana do Facebook é vazado revelando que a companhia monitorava em tempo real postagens, fotos e vídeos compartilhados por jovens para determinar quando estes usuários supostamente se sentiam ansiosos, bobos, fracassados, derrotados, nervosos. Elaborado como uma apresentação para um dos principais bancos australianos, o documento pretendia mostrar a capacidade da empresa de reunir informações psicológicas sobre uma numerosa base de dados: cerca de 1.9 milhão de estudantes de ensino médio, 1.5 milhão de universitários e três milhões de jovens trabalhadores (LEVIN, 2017).

Meses antes, em setembro de 2016, Alexander Nix, então diretor-executivo da consultora de marketing político Cambridge Analytica, profere palestra intitulada The Power of Big Data and Psychographics (O poder do Big Data e da psicometria, em português), no encontro anual da Concordia Summit, espécie de rendez-vous de empresários e políticos mundiais. Em tom autolaudatório e sem demonstrar pudor ou ressalvas aos limites éticos das ferramentas, Nix relata o trabalho desenvolvido pela empresa na campanha do senador Ted Cruz para influenciar e persuadir o eleitorado americano durante as primárias do partido republicano naquele ano (CONCORDIA, 2016). Nix buscava exaltar a eficácia da metodologia de publicidade direcionada desenvolvida pela Cambridge Analytica por meio da criação de perfis psicométricos a partir de dados pessoais e relacionais digitais, em comparação aos tradicionais métodos de análise e segmentação demográficos.

Os dois episódios compunham discretamente e sem maiores repercussões um já vasto repertório de usos controversos de dados pessoais digitais. Até que, em março de 2018, o New York Times (ROSENBERG; CONFESSORE; CADWALLADR, 2018) e o The Guardian (CADWALLADR; GRAHAM-HARRISON, 2018) publicam séries de matérias e reportagens, com base no depoimento e em documentos vazados por Christopher Wylie, um ex-funcionário da Cambridge Analytica, revelando que a empresa utilizou, indevidamente e sem o consentimento das pessoas envolvidas, dados de cerca de 87 milhões de perfis do Facebook para direcionar propaganda política em favor de Donald Trump durante as eleições presidenciais americanas de 2016. 
Até então, um dos episódios mais ruidosos sobre a utilização eticamente questionável de dados com efeitos persuasivos no comportamento humano ocorreu a partir de um experimento realizado pelo Facebook em 2014, cujos resultados foram publicados na revista científica Proceedings of the National Academy Sciences. Intitulado Evidência experimental de contágio emocional em escala massiva através de redes sociais (KRAMER; GUILLORY; HANCOCK, 2014), o artigo detalhou os resultados e conclusões da manipulação, ao longo de uma semana, do feed de notícias de quase 700 mil usuários, que foram divididos em dois grupos diferenciados pelo tipo de "conteúdo emocional" visualizado: o primeiro recebeu um filtro de conteúdos emocionalmente positivos e o segundo recebeu um filtro de conteúdos emocionalmente negativos durante este período.

Sem o conhecimento ou autorização dos envolvidos, o experimento tinha como propósito saber se o humor ou estado emocional desses grupos seria 'contaminado' pelo conteúdo visualizado no feed. Para tanto, as atualizações de status desses mesmos usuários foram monitoradas. Segundo os autores do artigo, a hipótese de contágio emocional teria sido confirmada pelo experimento. Ou seja, os usuários reproduziram, em suas atualizações de status, o estado emocional preponderante em seus feed.

Seguindo a mesma trilha dos rastros psicoafetivos e sociais, a via de acesso às informações de perfis do Facebook pela Cambridge Analytica foi, não por acaso, um teste de personalidade, chamado thisisyourdigitallife e ofertado como um aplicativo da rede social. No que consiste o tal teste de personalidade, que foi a isca mordida por 270 mil pessoas, dando acesso posteriormente, sem que elas soubessem, aos dados de sua rede de amigos na plataforma, alcançando informações de aproximadamente 87 milhões de perfis? Simplificadamente, o teste utilizado é baseado no modelo Big Five, que em psicometria consiste numa estrutura de cinco grandes fatores (extroversão, neuroticismo, socialização, realização e abertura à experiência) que remetem a dimensões de personalidade. Tais dimensões não representam um sistema teórico específico, e foram elaboradas a partir da análise dos termos que as pessoas usam, em linguagem natural, para definir a si mesmas e aos outros.

$\mathrm{O}$ teste baseia-se, assim, em um conjunto de questões (que pode variar de $20 \mathrm{a}$ 300) que avaliam as modulações dos cinco fatores que compõem a personalidade de uma pessoa, sendo um dos mais aceitos e replicados no mundo. O seu papel no caso da Cambridge Analytica é, contudo, relativamente secundário, pois os dados visados por esta empresa e outras similares não são tanto aqueles relativos a indivíduos específicos, baseados em um conhecimento profundo de suas personalidades. Ele funcionou, na verdade, como uma espécie de cavalo de Troia para acessar, minerar e explorar 
os dados relacionais, que são aqueles que interessam mais vivamente à economia psíquica dos algoritmos vigente no atual capitalismo de dados ${ }^{4}$, conforme veremos.

Por economia psíquica dos algoritmos (BRUNO, 2018) designamos o investimento contemporâneo - tecnocientífico, econômico e social - em processos algorítmicos de captura, análise e utilização de informações psíquicas e emocionais extraídas de nossos dados e ações em plataformas digitais (redes sociais, aplicativos, serviços de streaming, plataformas de compartilhamento e/ou consumo de conteúdo audiovisual etc.). As informações que interessam ao veloz capitalismo de dados não são mais apenas os rastros de nossas ações e interações (cliques, curtidas, compartilhamentos, visualizações, postagens), mas também sua "tonalidade" psíquica e emocional. É esta economia psíquica e afetiva que alimenta as atuais estratégias de previsão e indução de comportamentos nas plataformas digitais (e eventualmente fora delas).

Os exemplos que abrem esse artigo revelam, assim, não apenas os graves e indevidos usos de nossos dados pessoais para fins eleitorais e econômicos, mas explicitam os bastidores e as engrenagens de um capitalismo de dados cada vez mais feroz, e de um poderoso laboratório que, sob as interações online, captura, analisa e direciona imensos volumes de dados para aplicação de estratégias de modificação do comportamento humano. Eles não constituem, portanto, casos pontuais e isolados, mas sim uma nova lógica que entrelaça, de modo singular, corporações de tecnologia digital, ciência e sociedade.

No seio desta lógica, os dados pessoais digitais e suas informações psíquicas e emocionais são simultaneamente: a principal "moeda" do modelo de negócios que prevalece nas plataformas digitais; a fonte privilegiada de conhecimento de uma nova ciência de dados; um meio de controle do comportamento, orientado para diferentes fins, do consumo ao voto.

Essa tríplice característica dos dados psíquicos e emocionais constituem as três camadas da economia psíquica dos algoritmos que serão exploradas neste artigo: a camada propriamente econômica ou mercadológica; a camada epistemológica, voltada para a produção de conhecimento sobre indivíduos e populações; e a camada de gestão e controle comportamental. Notaremos que elas

\footnotetext{
4 Conforme veremos no próximo tópico deste artigo, o capitalismo de dados é definido como um sistema baseado na extração de valor e na mercantilização de dados digitais, perpassando as dimensões sociais, políticas e econômicas das redes sociotécnicas. Segundo West (2017), é um sistema no qual a comodificação de nossos dados engendra uma redistribuição assimétrica de poder, de modo a consolidar e fortalecer os atores que têm o acesso e a capacidade de dar sentido a tais informações. Tal noção também dialoga, em alguma medida, com as noções de capitalismo de vigilância, proposta por Shoshana Zuboff (2018) e de capitalismo de plataforma, formulada por Nick Srnicek (2017).
} 
se entrecruzam no cotidiano das plataformas e aplicativos digitais e assumem uma dimensão laboratorial extremamente inquietante.

As fronteiras entre o laboratório e a vida social, política e subjetiva tornam-se extremamente tênues. Estamos diante de um laboratório-mundo ou de uma ciência de plataforma 5 , intimamente conectados às engrenagens do mercado de dados pessoais, em que uma complexa e crescente economia psíquica e emocional nutre algoritmos que pretendem nos conhecer melhor do que nós mesmos, além de fazer previsões e intervenções sobre nossas emoções e condutas (BRUNO, 2018). Nos próximos tópicos, exploraremos cada uma das camadas mencionadas, enfatizando os cruzamentos entre elas, bem como o seu caráter laboratorial. Tal exploração está longe de ser exaustiva, mas pretende apontar elementos importantes para entender como processos psíquicos e emocionais despontam como fontes e alvos privilegiados de algoritmos que produzem valor, conhecimento e estratégias de gestão de nossos comportamentos online.

\section{Modelos de negócios, modelos epistemológicos: o valor dos dados psíquicos e emocionais}

Conforme apontamos, a atual dinâmica do capitalismo de dados ${ }^{6}$, centrado no modelo de negócios das plataformas e aplicativos digitais, tem como um de seus pilares a extração de valor de dados provenientes de mecanismos automatizados de coleta e análise de nossas ações e comportamentos online. Sob a ordem de grandeza do big data e a velocidade da gestão algorítmica, os difusos processos de monitoramento digital estão cada vez mais atrelados a estratégias econômicas que visam prever e modificar o comportamento humano.

O sucesso dessa lógica comercial está atrelado, segundo as análises de Zuboff (2018) sobre o que ela denomina "capitalismo de vigilância", a um certo modelo de publicidade e a práticas empresariais que teriam o Google como matriz. Eles

\footnotetext{
5 A expressão é uma referência ao já citado termo "Capitalismo de plataforma" (SRNICEK, 2017).

6 Sobre a noção de capitalismo de dados, conferir Nota 1, supra.

7 Matteo Pasquinelli (2015) aponta a criação do primeiro centro de processamento de dados do Google, conhecido como Google Cage, como o marco inicial do que ele conceitua como sociedade dos metadados. Segundo o autor, foi o primeiro banco de dados a operar de acordo com um mapeamento em escala global da topologia da internet e de suas tendências, de modo que "nos últimos anos, a sociedade em rede radicalizou uma mudança topológica: sob a superfície da rede, datacenters gigantescos foram transformados em monopólios de dados coletivos. Se as redes eram sobre fluxos abertos de informação (como Manuel Castells costumava defender), os datacenters são sobre o acúmulo de informações sobre informações, isto é, metadados. Além disso, sobre o algoritmo do Google, o PageRank, Pasquinelli (2010) afirma: "PageRank descreve especificamente o valor de atenção de qualquer objeto, a tal ponto que se tornou a principal e mais importante fonte de visibilidade e autoridade, mesmo fora da esfera digital".
} 
dependem da comercialização dos dados dos usuários a partir de processos conhecidos na linguagem publicitária como segmentação de mercado e microtargeting.

No entanto, a dinâmica em jogo nesta economia não se resume à venda de dados para publicidade, mas inclui também a venda do acesso em tempo real ao fluxo de ações online de indivíduos e populações que, através de ferramentas de análise algorítmicas, procuram influenciar e modificar os comportamentos a fim de gerar lucro (ZUBOFF, 2016).

Nesse sentido, mais importante do que o modelo de publicidade presente nestas plataformas é a promessa e capacidade de agir sobre os comportamentos enquanto eles acontecem. Por isso, queremos enfatizar a centralidade dos agenciamentos algorítmicos nas engrenagens de aplicativos e plataformas digitais. Seus processos de aprendizagem de máquina ${ }^{8}$ são responsáveis tanto pela produção de conhecimento a partir do imenso volume de dados gerados pelos usuários, quanto pela oferta personalizada de um mundo visível de ações e interações possíveis.

No cotidiano de plataformas digitais online, são coletados inúmeros tipos de informações de diferentes fontes. Nesta colheita, qualquer tipo de informação é relevante: desde "curtidas" do Facebook, passando pelas buscas no Google, e-mails, textos, fotos, músicas e vídeos, localizações, padrões de interações, redes, compras, movimentos, todos os cliques, até palavras com erros ortográficos, mensagens escritas e apagadas, velocidade de digitação, visualizações de páginas, e muito mais. Ainda que a escala do "big data" seja constituída por uma captura constante e ininterrupta de todo tipo de "small data" (ZUBOFF, 2018), desejamos ressaltar neste artigo o crescimento expressivo da relevância dos dados psíquicos e emocionais.

Além dos casos já citados, este interesse é visível, por exemplo, na popularização de ferramentas voltadas para a expressão e captura de emoções e estados psíquicos dos usuários em plataformas e aplicativos: emoticons, emojis, GIFs animados, stickers etc. Numa plataforma como o Facebook, podemos ver claramente a ampliação desse investimento: o primeiro passo explícito nessa direção se dá em 2009, com o lançamento do botão de "Curtir" (Like); em 2013, o espectro de expressão de emoções e estados psíquicos amplia-se enormemente com a opção de "Atualização de Status" (Status Update fields), permitindo que o usuário utilize uma grande diversidade de ícones gráficos para indicar a tonalidade

\footnotetext{
8 Nos referimos aqui às ferramentas de machine learning, que conferem aos códigos de um conjunto de algoritmos a capacidade de autoajuste e autocorreção, de modo a se adaptarem e revisarem seus resultados a partir de ações anteriores. O conjunto de algoritmos do feed do Facebook e do Page Rank do serviço de buscas do Google são exemplos: a cada interação na plataforma ou no buscador, seus algoritmos «aprendem» quais conteúdos seriam de maior relevância ou interesse dos usuários.
} 
emocional e psíquica de sua postagem. Na categoria "Sentimento/Atividade" o usuário pode escolher o sentimento que lhe é mais pertinente entre um leque de mais de 200 "carinhas" que correspondem a confiante, inspirado, esperançoso, frustrado, exausto, nostálgico, sexy etc. Em 2016, uma nova funcionalidade emocional passa a acompanhar o já banal botão "Like": os "Ícones de Reação" (Reaction Icons) permitem que qualifiquemos as postagens dos outros segundo um espectro de seis emoções básicas (Curtir, Amei, Haha, Uau, Triste e Grr).

Nota-se o quanto há todo um design e uma arquitetura voltados para alimentar algoritmos de plataformas e aplicativos com dados psíquicos e emocionais, de modo a torná-los disponíveis para o cálculo computacional. Além disso, como já apontamos, não são apenas os dados de cada um que importam aqui, mas, sobretudo, o seu valor relacional (GERLITZ; HELMOND, 2013). Assim, a relação entre as pessoas (LURY; DAY, 2019), bem como a interação entre seus processos emocionais e psíquicos se tornam disponíveis para o cálculo computacional, alimentando não apenas modelos de negócios das plataformas digitais, como também os modelos de conhecimento e gestão dos comportamentos.

Como se pode ver, trata-se tanto de uma mudança na estratégia de marketing e comércio de dados, quanto de um deslocamento histórico na produção de conhecimento sobre indivíduos e populações. Mais precisamente, trata-se de um saber que se exerce privilegiadamente sobre dividualidades ${ }^{9}$ e não tanto sobre indivíduos (DELEUZE, 1992; LAZZARATO, 2014; ROUVROY; BERNS, 2015). Por isso, no caso do Cambridge Analytica, os dados que mais interessam são aqueles que derivam das correlações entre os padrões de atividade dos usuários do Facebook e os perfis psicológicos - e não tanto os perfis psicológicos em si.

$\mathrm{O}$ aplicativo-teste thisisyourdigitallife, utilizado pela consultora de marketing político, foi elaborado com base no myPersonality, um outro aplicativo desenvolvido e utilizado entre 2007 e 2012 pelos pesquisadores da Cambridge University, Michal Kosinski e David Stilwell, para coletar e analisar dados de usuários do Facebook neste caso, com o consentimento dos mesmos. Também estruturado a partir do modelo dos cinco fatores descrito anteriormente, este segundo aplicativo oferecia aos usuários um teste de personalidade e, em troca, recebia a autorização para capturar e utilizar, para fins de pesquisa acadêmica, os dados de seus perfis psicológicos (acessado via teste) e dos seus próprios perfis do Facebook.

\footnotetext{
9 Referimo-nos ao conceito empregado por Deleuze (1992) para caracterizar os processos de subjetivação característicos das sociedades de controle, que deixam de operar predominantemente com a norma disciplinar e a produção de individualidade(s) para privilegiar a modulação de diferenças e a condução das condutas através do reconhecimento de padrões de componentes parciais da subjetividade.
} 
Com base no modelo dos pesquisadores da Cambridge University, o teste de personalidade utilizado pela Cambridge Analytica visa menos um conhecimento individualizado, unificado e aprofundado da personalidade de indivíduos específicos do que um conhecimento sobre as correlações entre os diferentes traços de personalidade e de atividade de inúmeros perfis. Assim, o conhecimento dessas correlações pretende revelar padrões supraindividuais ou interindividuais que permitam fazer predições em larga escala.

Nesse sentido, o que o caso do Cambridge Analytica nos revela sobre a dinâmica mais ampla do capitalismo de dados é que o teste de personalidade realizado por um indivíduo permitiria prever a personalidade de muitas outras pessoas similares a ele. Essa similaridade, vale dizer, concerne a "parcelas" de seu perfil de atividade e de personalidade - como, por exemplo, a correlação entre seu grau de extroversão (indicado no teste de personalidade) e o seu número de amigos e a frequência com que atualiza seu status e interage em grupos no Facebook (indicados no seu padrão de atividade)..$^{\circ}$ Ou entre o seu nível de socialização indicado no teste de personalidade e a frequência com que aparece em fotos com outros usuários." Um conhecimento sobre a correlação entre traços parciais (dividuais, portanto), e não tanto sobre indivíduos considerados em sua unidade.

Ao mesmo tempo, como vimos, esse saber extraído das correlações entre dados parciais de nossos perfis e condutas visa orientar conteúdos cada vez mais específicos e pertinentes a indivíduos particulares. Ou seja, um saber extraído de dados parciais e relacionais geraria uma "inteligência" que se pretende preditiva sobre alvos individuais cada vez mais precisos (em especial, como invocado na fala de Alexander Nix citada na introdução, com uma ênfase maior na segmentação psicométrica do que na demográfica). Vemos, portanto, que se trata de um conhecimento que opera sobre uma dupla escala - dividual e individual - visando inferir algoritmicamente a personalidade de pessoas que se tornarão objetos futuros de diferentes tipos de ações (microtargeting, sistemas de recomendação, direcionamento de conteúdos etc.). Neste sentido, esta condução algorítmica de condutas pode não levar em conta sujeitos específicos, mas não deixa de mirar alvos (ROUVROY; BERNS, 2015).

\footnotetext{
10 Ao analisar a correlação entre o teste de personalidade de um grupo de usuários e seus padrões de atividades no Facebook, pretende-se, fazendo o caminho "inverso", inferir ou "prever" a personalidade de muitos outros usuários a partir dos seus padrões de atividade.

"Tal frequência pode ser mensurada pelo número de vezes que o usuário é "marcado" em fotos de outras pessoas. Presente no Facebook e também em outras plataformas digitais, o mecanismo de "marcar" ou de "tags" diz respeito ao recurso técnico que permite associar o conteúdo de uma imagem ou postagem, a partir de um hiperlink, ao perfil de um usuário específico.
} 
O saber preditivo dos algoritmos define, deste modo, os perfis de alvos específicos para sugestão de conteúdos diferenciados no momento e no contexto apropriados para influenciar, de forma personalizada e em tempo real, o comportamento dos usuários (INTRONA, 2016). Legitimadas por um discurso de comodidade que promete oferecer conteúdos, serviços e produtos ultrapersonalizados e "relevantes" aos interesses dos usuários, estas ferramentas de captura irrestrita e extensiva de informações são, entretanto, entendidas pelo marketing digital como meios de explorar vulnerabilidade cognitivas e emocionais a fim de influenciar o processo de tomada de decisão e o comportamento dos usuários.

Um exemplo disso, segundo Nadler e McGuigan (2017), consiste nas estratégias de marketing digital e de design de plataforma que têm se apropriado da linguagem e das técnicas da Economia Comportamental, reunindo um conjunto de teorias do campo psicológico - tais como o behaviorismo, a psicologia cognitiva, psicologia evolutiva e a neuropsicologia - para desenvolver modelos que buscam identificar e prever padrões da forma como as pessoas tomam decisões econômicas, de modo a intervir sobre essas escolhas. Nas aplicações computacionais que têm essas teorias como modelo epistemológico, os usuários não são concebidos como consumidores racionais e perfeitamente informados, mas sim como impulsivos e "previsivelmente irracionais"."2

Assim, fatores contextuais e tendências cognitivas são explorados para construir o que os economistas comportamentais chamam de "arquitetura de decisões", isto é, uma organização específica do contexto no qual as decisões são tomadas a fim de influenciar a ação das pessoas em certa direção. Tais técnicas podem envolver desde a elaboração da interface, o design de softwares, os recursos técnicos das próprias plataformas, até os sistemas de recomendação.

Fica mais claro, a essa altura, de que modo se entrecruzam as três camadas que retroalimenta a economia psíquica dos algoritmos. Processos automatizados de captura, análise e utilização de dados psíquicos e emocionais estão na base de um modelo de negócios que é inseparável de modelos específicos de conhecimento sobre a cognição e o comportamento humanos que, por sua vez, estão atrelados a estratégias de gestão de condutas, como veremos no próximo tópico.

12 Previsivelmente irracional", no original em inglês Predictably Irrational, se refere ao do livro do economista comportamental Dan Ariely, que se tornou uma das referências mais populares e influentes no uso deste tipo de abordagem aplicada ao marketing digital e ao design de softwares (SEAVER, 2018). 


\section{Gestão algorítmica da conduta: da previsão à captura}

A breve história do monitoramento de rastros digitais na internet sempre esteve relacionada a mecanismos de intervenção sobre as condutas online (BRUNO, $2012 \mathrm{e}$ 2013). Apesar da diversidade de dispositivos e aplicações dos sistemas automatizados de monitoramento e análise dos rastros digitais, podemos afirmar que o modelo preditivo prevaleceu na última década (BRUNO, 2013; ZUBOFF, 2018). Conformejá apontamos, tal modelo consiste em mecanismos automatizados de captura, processamento e análise do maior volume e diversidade possível de dados, buscando extrair padrões que orientam previsões e consequentemente intervenções sobre comportamentos futuros. Esse paradigma é patente na conclusão do artigo sobre a pesquisa realizada com base no teste myPersonality, mencionada no tópico anterior:

[...] o estudo mostra que, combinando várias características, podemos fazer previsões relativamente precisas em relação à personalidade de um indivíduo, sendo a Extroversão a mais fácil de prever e a Socialização sendo a mais elusiva. Uma aplicação potencial para o nosso trabalho é a publicidade online e os sistemas de recomendação. Analisando as informações das redes sociais, seria possível "perfilar" os indivíduos, dividindo automaticamente os usuários em diferentes segmentos e adaptando os anúncios a cada segmento com base na personalidade. Da mesma forma, pode-se imaginar a construção de sistemas de recomendação baseados em perfis de personalidade (BACHRACH e outros, 2012, p. 31, tradução nossa).13

Não poderia ser mais clara a sobreposição das três camadas da economia psíquica dos algoritmos, assim como a centralidade do modelo preditivo, que reúne num só golpe promessas de precisão científica, aplicação comercial e intervenção sobre o comportamento daqueles que são simultaneamente objetos de estudo, usuários e clientes potenciais. Entretanto, tal centralidade começa a ser perturbada ou disputada por outro modelo: o da captura ou do engajamento.

Por que gastar tempo e inteligência computacional prevendo comportamentos se as plataformas e aplicativos permitem intervenções em tempo real sobre

13 Livre tradução para: "[...] the study shows that by combining several features, we can make relatively accurate predictions regarding a individual's personality, with Extraversion being most easy to predict and Agreeableness being most elusive. One potential application for our work is online advertising and recommender systems. By analysing information from social networks it would be possible to "profile" individuals, automatically dividing users into different segments, and tailor advertisements to each segment based on personality. Similarly, one can imagine building recommender systems based on personality profiles". 
a conduta dos usuários? De uma certa perspectiva, podemos ver o modelo da captura/engajamento como uma espécie de aceleração do modelo preditivo: o aumento da capacidade e velocidade de monitoramento e processamento em tempo real das ações dos usuários online torna dispensável a previsão, permitindo que os algoritmos atuem de modo ainda mais performativo do que no modelo preditivo, intervindo no próprio fluxo das condutas enquanto elas acontecem.

Não se trata, entretanto, apenas de uma proposta mais veloz. Há mudanças significativas nas estratégias de gestão e controle dos comportamentos.

Tais mudanças podem ser observadas na trajetória dos sistemas de recomendação algorítmicos, ferramenta que vem se espraiando e adquirindo protagonismo na mediação da oferta de conteúdo cultural, comercial e político por diversas plataformas. ${ }^{14}$ Nos últimos anos, desenvolvedores digitais têm se voltado cada vez mais para a arquitetura e o design desses sistemas, visando não apenas prever preferências, interesses e comportamentos futuros, mas sobretudo capturar, enganchar e engajar a atenção de usuários.

Nessa mudança de um paradigma preditivo para um paradigma de captura, tais ferramentas algorítmicas funcionam cada vez mais com o objetivo de manter os usuários o máximo de tempo conectados às plataformas digitais. Enquanto no primeiro "um sistema de recomendação prevê como os usuários avaliarão os itens e é julgado pela precisão de suas previsões"15 (SEAVER, 2018, p.10, tradução nossa), tendo como contraprova de acerto e de satisfação a avaliação explícita dos usuários (likes, número de estrelas ou notas de avaliação, por exemplo), o segundo tem como premissa que "ser preciso não é suficiente" (MCNEE; RIEDL; KONSTAN, 2006) e que a eficiência de um sistema de recomendação é medida pela capacidade em capturar a atenção e produzir o engajamento dos usuários (BENTES, 2019).

Este movimento que Seaver denomina de virada captológica (captological turn) tem como principal referência o trabalho desenvolvido por B.J Fogg, fundador do Persuasive Technology Lab e criador do campo de pesquisa que ele designou por "captology", termo derivado da sigla em inglês de computers as persuasive technologies. Ligado à Universidade de Stanford, Fogg é um dos precursores no desenvolvimento de modelos e métodos no campo do "behavioral design", que combina teorias da psicologia behaviorista com a psicologia cognitivo-compor-

14 Netflix, Tinder e Spotfy são exemplos, dentre inúmeras outras, de plataformas que investem fortemente em sistemas de recomendação.

15 Livre tradução para: «a recommender system predicts how users will rate items, and it is judged by how accurate its predictions were.» 
tamental, a economia comportamental e as neurociências em aplicações para a economia digital e a indústria computacional.

De acordo com a apresentação no site do laboratório, o objetivo dessas técnicas e saberes é "criar respostas sobre como produtos de computação - de sites a softwares de smartphones - podem ser projetados para alterar crenças e comportamentos" (STANFORD PERSUASIVE TECH LAB, 2019, tradução nossa). ${ }^{16}$

Vemos, portanto, que essa virada não implica apenas uma mudança no funcionamento desses sistemas, mas também um deslocamento de como são percebidos, conhecidos e operados a satisfação e o desejo das pessoas, bem como suas crenças e comportamentos. As plataformas de recomendação e de sociabilização deixam, assim, de privilegiar formas explícitas de avaliação feitas pelos próprios usuários. Em vez disso, passam a valorizar tipos de métricas implícitas e a sua tonalidade psíquica e emocional como evidências dos juízos e preferências das pessoas.

Por exemplo, ao invés de priorizar notas de classificações, comentários, compartilhamentos, tornam-se cada vez mais relevantes para o funcionamento desses sistemas certas informações como o tempo médio gasto em tipos diferentes de postagens, a pausa em um vídeo, o padrão de navegação, pular uma música recomendada ou um determinado conteúdo.

Essas mudanças nas estratégias de gestão algorítmica da conduta estão diretamente ligadas ao modelo de negócios das plataformas digitais, como mencionado anteriormente. Para a expansão deste mercado de dados, uma condição é fundamental: que os usuários gastem o maior tempo possível em plataformas ou dispositivos, para, assim, seus dados serem extraídos e seus comportamentos se tornarem reconhecíveis e suscetíveis a intervenções.

Nesse sentido, a atual dinâmica do capitalismo de dados está intimamente ligada aos já conhecidos mecanismos de uma economia da atenção (GOLDHABER, 1997; DAVENPORT; BECK, 2001). Sua premissa fundamental é a de que, em meio a um espaço-tempo cada vez mais saturado de estímulos visuais e informacionais (CRARY, 2014), a atenção se torna um recurso escasso e, por sua vez, extremamente valioso e imensamente disputado (CALIMAN, 2012; CITTON, 2016; WU, 2016).

Não por acaso, como vimos, a disputa econômica pela atenção vem popularizando um tipo de abordagem na indústria tecnológica que aposta no desenvolvimento de uma arquitetura de decisões nas plataformas direcionada para capturar e cativar a atenção dos usuários, explorando tendências e vulnerabilidades

16 Tradução livre de: "creates insight into how computing products — from websites to mobile phone software - can be designed to change what people believe and what they do". 
cognitivas que mantenham os usuários enganchados e engajados nesses serviços. Engendrando, assim, uma lógica circular entre a experimentação da dimensão laboratorial e os efeitos sociais, políticos e subjetivos do capitalismo de dados.

Por isso, um dos principais objetivos das empresas de tecnologia no paradigma da captura é fazer com que o uso de seus serviços não seja apenas um comportamento pontual, mas se torne um hábito. Entendido, nesse contexto tecnobehaviorista do design e da arquitetura das plataformas, como "comportamentos automáticos desencadeados por pistas situacionais: coisas que fazemos com pouco ou nenhum pensamento consciente" (EYAL, 2014, p.8), o hábito é construído por pequenos estímulos e recompensas. Tais comportamentos são assim incentivados a se tornar uma prática rotineira, de modo que usuários retornem constantemente a esses serviços e preferencialmente nunca saiam deles.

\section{Considerações finais}

O problema das modernas teorias do behaviorismo não é que estejam erradas, mas sim que possam vir a tornarem-se verdadeiras, que realmente constituam as melhores conceituações possíveis de certas tendências óbvias da sociedade moderna (ARENDT, 1999, p. 336).

A captura e manutenção dos usuários nessas plataformas garante, num mesmo movimento, a alimentação, testagem e aprimoramento contínuos do que estamos propondo chamar de laboratório de plataforma. A atividade constante dos usuários alimenta a aplicação de técnicas de aprendizagem de máquina e de inteligência artificial, aprimorando as operações algorítmicas que, por sua vez, atuam sobre os usuários, criando um ciclo que retroalimenta as três camadas da economia psíquica dos algoritmos, reforçando a sua dimensão laboratorial.

Ainda conhecemos muito pouco desses laboratórios que, no entanto, estão cada vez mais misturados ao nosso cotidiano. Os muros dos tradicionais e purificados laboratórios científicos e psicométricos dão lugar a caixas pretas digitais bastante opacas. O laboratório ganha mundo, coletando dados e sujeitos "in the wild"17, mas segundo uma dinâmica extremamente assimétrica. Enquanto os sujeitos ${ }^{18}$, seus comportamentos e suas emoções se tornam cada vez mais

17 A expressão, na língua inglesa, designa uma condição natural e independente da intervenção humana. Coloquialmente, também designa no "mundo real", em oposição a um mundo teorizado ou imaginado.

18 Na linguagem e protocolo da ciência experimental, os "sujeitos" são aqueles que participam de um experimento científico. 
acessíveis aos atores humanos e não humanos das corporações que operam esses laboratórios, estes são muito pouco inteligíveis para aqueles que são seus "usuários" e suas fontes de conhecimento e pesquisa.

Como chama atenção Zuboff (2019), no capitalismo de vigilância, a experiência humana é tomada como matéria-prima disponível e acessível gratuitamente a um tipo de poder que não apenas automatiza o fluxo de informações sobre nós, mas visa automatizar nosso próprio comportamento. Não à toa, o denunciante Chris Wylie, em sua declaração ao The Guardian (CADWALLADR, 2018), referiu-se diversas vezes ao caso da Cambridge Analytica e Facebook como um "experimento" em que se tratava de elaborar "armas psicológicas" para uma "guerra cultural cujo campo de batalha seria a internet (as mídias sociais) e o alvo, cada um de nós".

Quando nos perguntamos sobre o grau de eficácia ou confiabilidade das técnicas e experimentos conduzidos por tais laboratórios, seguimos desconhecendo grande parte do problema. Sabemos que as ciências comportamental e psicométrica - nas bases dessas pesquisas e experimentos sobre dados psíquicos, emocionais e atencionais - estão longe de ser consensuais. Os programas de conhecimento e de intervenção sobre indivíduos e populações que se atualizam nesse estranho laboratório de plataforma já acumulam cerca de um século de críticas em diversos campos: na própria psicologia e em certos ramos das ciências cognitivas, como também na sociologia e na filosofia (ROSE, 1990). Além disso, estão longe de ser consistentemente fiáveis as promessas de previsão de personalidade e de orientação psíquico-comportamental efetuadas tanto pelas corporações quanto por alguns artigos científicos.

A leitura de um trecho das conclusões de uma das pesquisas tomadas como referência para empresas como a Cambridge Analytica e similares é suficiente para interrogar o quanto suas conclusões talvez sejam superestimadas:

Mostramos que registros digitais facilmente acessíveis de comportamento, Facebook Likes, podem ser usados para prever automaticamente e com precisão uma variedade de atributos pessoais altamente sensíveis, incluindo orientação sexual, etnia, visões religiosas e políticas, traços de personalidade, inteligência, felicidade e uso de substâncias aditivas, separação parental, idade e sexo (KOSINSKI, STILLWELL, GRAEPEL, 2013, p.5082, tradução nossa). ${ }^{19}$

\footnotetext{
19 Livre tradução para: "We show that easily accessible digital records of behavior, Facebook Likes, can be used to automatically and accurately predict a range of highly sensitive personal attributes including: sexual orientation, ethnicity, religious and political views, personality traits, intelligence, happiness, use of addictive substances, parental separation, age, and gender".
} 
O caráter controverso da alegada acuidade das previsões algorítmicas não chega, contudo, a ser um alento. $O$ fato de tais pesquisas e estratégias estarem sujeitas a muitas falhas e erros não minimiza as nossas inquietações. Os erros não significam, neste contexto, ausência de efeitos. A descoberta de padrões por indução estatística, base da análise algorítmica, não exclui de seu aprendizado os desvios e anomalias (PASQUINELLI, 2015).

Retomando o caso do experimento de contágio emocional, citado anteriormente, vale enfatizar que, apesar de afirmarem que a hipótese foi comprova$\mathrm{da}$, os resultados foram estatisticamente baixos, cientificamente irrelevantes, poderíamos dizer. Segundo o artigo (KRAMER, GUILLORY e HANCOCK, 2014), quando postagens positivas foram reduzidas no News Feed, a porcentagem de palavras positivas nas postagens das pessoas diminuiu $0,1 \%$, enquanto o percentual de palavras negativas aumentou $0,04 \%$. Já quando os posts negativos foram reduzidos, as palavras negativas postadas por usuários diminuíram em 0,07\%, e o número de palavras positivas aumentou $0,06 \%$.

Entretanto, um aspecto citado no artigo de modo secundário, sem maiores detalhes, como uma espécie de "efeito colateral" do experimento nos dá importantes pistas sobre a mudança do interesse das empresas por dados psíquicos e emocionais e sobre o deslocamento do paradigma preditivo para o da captura/engajamento: notou-se que a exposição dos usuários a conteúdos emocionais, tanto positivos quanto negativos, os tornou mais ativos e engajados na plataforma. Este resultado nos aponta que os processos de gestão algorítmica de dados psíquicos e emocionais podem produzir efeitos e consequências na modulação e controle do comportamento das pessoas mesmo sem inferir necessariamente atributos como "personalidade" ou "identidade".

Nesse caso, vemos bem como a modulação e o controle do comportamento da economia psíquica dos algoritmos são exercidos privilegiadamente através de alterações no contexto e no ambiente da oferta de conteúdo, propiciados pela arquitetura e o design das plataformas. Embora alimentada por dados psíquicos e emocionais, o valor dessa economia psíquica dos algoritmos não deriva propriamente da acuidade de previsões de personalidade, mas sim da capacidade de intervir em tempo real nas ações e emoções dos usuários.

O laboratório aqui em questão é, sobretudo, performativo. Numa base gigantesca de conteúdos direcionados para centenas de milhões de "alvos", mesmo uma margem relativamente baixa de acertos já é bastante alta se comparada aos métodos tradicionais de propaganda direcionada. Como reconhecem os autores do experimento de contágio: "dada a escala maciça de redes sociais 
como o Facebook, mesmo pequenos efeitos podem ter grandes consequências" (KRAMER; GUILLORY; HANCOCK, 2014, tradução nossa). ${ }^{20}$

Assim, a escala e o ecossistema dessas plataformas laboratórios são bastante tolerantes às falhas e seus resultados podem ser testados de modo quase ininterrupto e em tempo quase real. Os erros não invalidam seus testes, experimentos e aplicações, ao contrário, também alimentam os algoritmos e suas bases de dados, permitindo revisões relativamente rápidas dos procedimentos e das tomadas de decisão em curso. Dessa forma, com todos os limites e falhas, as implementações de tais pesquisas no cotidiano de nossas vidas podem ser entendidas, sem maiores exageros, como experimentos corporativo-científicos com implicações a um só tempo mercadológicas, sociais, subjetivas e políticas.

Cabe ainda destacar que a alardeada eficácia de tais experimentos, anunciados como capazes de descobrir, graças à inteligência algorítmica, padrões em uma escala de informações que a cognição humana não alcança, não deve mascarar os processos discursivos e contingências materiais e sociotécnicas que os legitimam (REIGELUTH, 2014, p. 244). A própria escolha de modelos simplificados de análise de personalidade, como o Big Five, se dá nesse contexto não tanto pela acuidade, como citado, mas pelo fato de serem mais facilmente legíveis e interpretadas por processos maquínicos.

O desenvolvimento desses programas requer uma escrita ou linguagem que seja, ao mesmo tempo, legível para os padrões e lógicas da máquina, como também operável para a tradução de outputs interpretáveis por humanos. Um diagnóstico similar é proposto pelo artista e pesquisador Ruben van de Ven (2017), em seu estudo de sistemas automatizados de análise de emoções a partir os rostos humanos: a utilização e legitimação dessas tecnologias seria menos capaz de aferir os estados emocionais das pessoas do que de normalizar o que se entende por emoções como "raiva", "tristeza" e "desprezo". Ao invés de "dar novos insights sobre como os seres humanos interagem, esses sistemas reforçam uma pré-concepção existente do que são as emoções. Por essa razão, a tecnologia fornece uma diretriz para os humanos se expressarem". ${ }^{21}$

As inquietações que daí derivam não são apenas sobre ciência mal aplicada, ou sobre negócios e propagandas, nem só sobre vigilância e privacidade. É sobre fabricação de mundos. O que está em jogo é uma economia psíquica dos algoritmos

\footnotetext{
20 Livre tradução para: "[...] given the massive scale of social networks such as Facebook, even small effects can have large aggregated consequences".

${ }^{21}$ Livre tradução para: "Rather than giving new insights into how humans interact, these systems reinforce an existing preconception of what emotions are. For that reason, the technology ultimately provides a guideline for humans to express themselves".
} 
que, com suas estratégias próprias, extrai valor e capitaliza nossa atenção, nossos estados psíquicos e afetivos a fim de produzir efeitos reais nas paisagens de dados e informações por onde trafegamos, em nossa percepção e em nossas condutas.

\section{REFERÊNCIAS}

ARENDT, Hannah. A condição humana. Rio de Janeiro: Forense Universitária, 1999.

BACHRACH, Yoram; KOSINSKI, Michal; GRAEPEL, Thore; KOHLI, Pushmeet, STILLWELL, David. Personality and patterns of Facebook usage. Proceedings of the 4th Annual ACM Web Science Conference. ACM, jun. 2012. Disponível em: https://www.microsoft.com/ en-us/research/publication/personality-and-patterns-of-facebook-usage/. Acesso em: 17 mar. 2018. https://doi.org/10.1145/2380718.2380722

BENTES, Anna. A gestão algorítmica da atenção: enganchar, conhecer e persuadir. In: POLIDO, Fabrício B. P.; ANJOS, Lucas Costa dos; BRANDÃO, Luiza C. C. (org.). Políticas, internet e sociedade. Belo Horizonte: IRIS, 2019.

BRUNO, Fernanda. Rastros digitais sob a perspectiva da teoria ator-rede. Revista FAMECOS (Online), Porto Alegre, v. 19, p. 681, 2012. https://doi.org/10.15448/19803729.2012 .3 .12893

BRUNO, Fernanda. Máquinas de ver, modos de ser: vigilância, tecnologia, subjetividade. Porto Alegre: Sulina, 2013.

BRUNO, Fernanda. A economia psíquica dos algoritmos: quando o laboratório é o mundo. NEXO Jornal, Brasil, p. 1-3, 12 jun. 2018.

CADWALLADR, Carole. 'I made Steve Bannon's psychological warfare tool': meet the data war whistleblower. The Guardian. 18 mar. 2018. Disponível em: <https://www. theguardian.com/news/2018/mar/17/data-war-whistleblower-christopher-wylie-faceook-nix-bannon-trump> Acesso em 17 de março de 2018. Acesso em: 27 mar. 2018.

CADWALLADR, Carole; GRAHAM-HARRISON, Anne. Revealed: 50 million Facebook profiles harvested for Cambridge Analytica in major data breach. The Guardian. 17 mar. 2018. Disponível em: https://www.theguardian.com/news/2018/mar/17/cambridge-analytica-facebook-influence-us-election. Acesso em: 27 mar. 2018.

CALIMAN, Luciana. Os regimes da atenção na subjetividade contemporânea. Arq. bras. psicol., Rio de Janeiro, v. 64, n. 1, p. 02-17, abr., 2012.

CITTON, Yves. The ecology of attention. Malden: Polity Press, 2016.

CONCORDIA. Cambridge Analytica - The Power of Big Data and Psychographics. 2016. Disponível em: https://www.youtube.com/watch?v=n8Dd5aVXLCc. Acesso em: 18 mar. 2018. 
CRARY, Jonathan. 24/7 - Capitalismo e os fins do sono. São Paulo: Contraponto, 2014. https://doi.org/10.1590/1517-106x/183-581

DAVENPORT, Thomas; BECK, John C. A economia da atenção. Rio de Janeiro: Campus, 2001.

DELEUZE, Gilles. Pos-Scriptum: Sobre as sociedades de controle. Conversações, 19721990. Rio de Janeiro: Ed. 34, 1992.

EYAL, Nir. Hooked: How to Build Habit-forming Products. Nova York: Portfolio/Penguin, 2014.

GERLITZ, Carolin; HELMOND, Anne. The like economy: Social buttons and the data-intensive web. New media \& society, v. 15, n. 8, p. 1348-1365, 2013. https://doi. org/10.1177/1461444812472322

GOLDHABER, Michael H. The Attention Economy and the Net. First Monday, v. 2, n. 4, 07 abr. 1997. Disponível em: https://firstmonday.org/article/view/519/440. Acesso em: 15 dez. 2017.

GRASSEGGER, Hannes; KROGERUS, Mikael. Os dados que viraram o mundo de cabeça para baixo. Motherboard. 13 fev. 2017. Disponível em: https://www.vice.com/pt_br/ article/8qkgyp/os-dados-que-viraram-o-mundo-de-cabeca-para-baixo. Acesso em: 19 mar. 2018. https://doi.org/10.17771/pucrio.acad.5117

INTRONA, Lucas. The Algorithmic choreography of the impressionable subject. In: SEYFERT, R.; ROBERGE, J. Algorithmic Cultures: essays on meaning, performance and new Technologies. Nova York: Routledge, 2016. https://doi.org/10.4324/9781315658698

KOSINSKI, Michal; STILLWELI, David; GRAEPEL, Thore. Private traits and attributes are predictable from digital records of human behavior. Proceedings of the National Academy of Sciences, v. 110, n. 15, p. 5802-5805, abr. 2013. Disponível em: https:// www.pnas.org/content/pnas/110/15/5802.full.pdf. Acesso em: 18 mar. 2018. https:// doi.org/10.1073/pnas.1218772110

KRAMER, Adam D. I.; GUILLORY, Jamie E.; HANCOCK, Jeffrey T. Experimental evidence of massive-scale emotional contagion through social networks. Proceedings of the National Academy of Sciences, jun., 2014. Disponível em: http://www.pnas.org/content/111/24/8788.full. Acesso em: 15 mar. 2017. https://doi.org/10.1073/pnas.1320040111

LAZZARATO, Maurizio. Signos, máquinas, subjetividades. São Paulo; Helsinque: n. 1; Edições Sesc São Paulo, 2014.

LEVIN, Sam. Facebook told advertisers it can identify teens feeling 'insecure' and 'worthless'. The Guardian, maio 2017. Disponível em: https://www.theguardian.com/ technology/2017/may/01/facebook-advertising-data-insecure-teens. Acesso em: 18 mar. 2018. 
MCNEE, Sean M., RIEDLI, John; KONSTAN, Joseph A. Being Accurate Is Not Enough: How Accuracy Metrics Have Hurt Recommender Systems. CHI'06 Extended Abstracts on Human Factors in Computing Systems, 1097-1101. ACM, 2006. Disponível em: http://dl.acm.org/citation.cfm?id=1125659. Acesso em: 18 mar. 2018. https://doi. org/10.1145/1125451.1125659

LURY, Celia; DAY, Sophie. Algorithmic personalization as a mode of individuation. Theory, Culture \& Society, v. 36, n. 2, p. 17-37, 2019. https://doi.org/10.1177/0263276418818888

NADLER, Anthony; MCGUIGAN, Lee. An impulse to exploit: the behavioral turn in data-drive marketing. Critical Studies in Media Communication, out., 2017. https://doi. org/10.1080/15295036.2017.1387279

PASQUINELLI, Mateo. Um diagrama do capitalismo cognitivo e da exploração da inteligência social geral. 16 de março de 2010. Disponível em: http://matteopasquinelli. com/docs/Pasquinelli PageRank.pdf. Acesso em: 10 abr. 2018.

PASQUINELLI, Mateo. Anomaly Detection: The Mathematization of the Abnormal in the Metadata Society, 2015. Disponível em:

https://www.academia.edu/10369819/Anomaly_Detection_The Mathematization of the Abnormal in the Metadata Society. Acesso em: 1 maio 2018.

REIGELUTH, Tyler. Why data is not enough: Digital traces as control of self and selfcontrol. Surveillance \& Society, n. 12, v. 2, p. 243-354, 2014. https://doi.org/10.24908/ Ss.v12i2.4741

ROSE, Nikolas. Governing the soul: the shaping of the private self. Londres: Taylor \& Francis/Routledge, 1990.

ROSEMBERG, Matthew; CONFESSORE, Nicholas; CADWALLADR, Carole. How Trump Consultants Exploited the Facebook Data of Millions. The New York Times. 17 mar. 2018. Disponível em: https://www.nytimes.com/2018/03/17/us/politics/cambridge-analytica-trump-campaign.html. Acesso em: 27 mar. 2018.

ROUVROY, Antoinette; BERNS, Thomas. "Governamentalidade algorítmica e perspectivas de emancipação: o díspar como condição de individuação pela relação?". Revista Eco-Pós (Online), v. 18, p. 36-56, 2015.

SEAVER, Nick. Captivating algorithms: recommender systems as traps. Journal of Material Culture, ago., 2018.

SEYFERT, Robert; ROBERGE, Jonathan. Algorithmic Cultures: essays on meaning, performance and new Technologies. Nova York: Routledge, 2016.

SRNICEK, Nick. Platform Capitalism. Malden: Polity Press, 2017 
STANFORD PERSUASIVE TECH LAB. Welcome to the lab. Página inicial. 2019. Disponível em: http://captology.stanford.edu/. Acesso em: 15 dez. /2017.

VEN, Ruben van de. Choose How You Feel; You Have Seven Options. Institute of network cultures, jan. 2017. Disponível em: http://networkcultures.org/longform/2017/01/25/ choose-how-you-feel-you-have-seven-options/. Acesso em: 19 jun. 2018. https://doi. org/10.3726/978-3-0353-0219-6/10

WU, Tim. The attention merchants: the epic scramble to get inside our heads. Nova York: Knopf, 2016.

ZUBOFF, Shoshana. Secrets of Surveillance Capitalism. Frankfurter Allgemeine, 5 mar. 2016. Disponível em: http://www.faz.net/aktuell/feuilleton/debatten/the-digital-debate/ shoshana-zuboff-secrets- of-surveillance-capitalism-14103616.html?printPagedArticle=true\#pagelndex_2. Acesso em: 4 abr. 2018. https://doi.org/10.24908/ss.v17i1/2.13238

ZUBOFF, Shoshana. Big Other: Capitalismo de vigilância e perspectivas para uma civilização de informação. In: BRUNO, Fernanda; CARDOSO, Bruno; KANASHIRO, Marta; GUILHON, Luciana; MELGAÇO, Lucas (org.). Tecnopolíticas da vigilância: perspectivas da margem. São Paulo: Boitempo, 2018.

https://doi.org/10.11606/d.7.2007.tde-12062007-150511

ZUBOFF, Shoshana. The Age of Surveillance Capitalism: The Fight for a Human Future at the New Frontier of Power. Londres: Profile Books, 2019.

\section{Dados dos autores:}

Fernanda Glória Bruno - e-mail: bruno.fernanda@gmail.com

Professora do Programa de Pós-Graduação em Comunicação e Cultura da UFRJ.

Anna Carolina Franco Bentes - e-mail: annacbentes@gmail.com

Doutoranda do Programa de Pós-Graduação em Comunicação e Cultura da UFRJ.

Paulo Faltay - e-mail: pfaltay@gmail.com

Doutorando do Programa de Pós-Graduação em Comunicação e Cultura da UFRJ.

\section{Endereço dos autores:}

Universidade Federal do Rio de Janeiro, Programa de Pós-Graduação em Comunicação e Cultura - Av. Pasteur n² 250 - fds, Urca - Rio de Janeiro (RJ), Brasil 